\title{
Simulation of Pulse propagation in optical fibers
}

\author{
P. C. T. Munaweera, K.A.I.L. Wijewardena Gamalath \\ Department of Physics, University of Colombo, Colombo3, Sri Lanka \\ e-mail: imalie@phys.cmb.ac.lk
}

Keywords: Optical fibers, self-phase modulation, group velocity dispersion, Gaussian pulse, hyperbolic secant pulse, soliton, split step Fourier method.

\begin{abstract}
A theoretical model was developed for light pulses propagating in optical fibers by considering the nonlinear effects, the self-phase modulation and group velocity dispersion effects. The split step Fourier method was used to generate soliton pulses in a fiber composed of a glass core surrounded by a cladding layer. Gaussian and hyperbolic secant input pulses were used for the simulation. By varying the initial chirp, input power and nonlinear coefficient for an input Gaussian pulse at wavelength of $\lambda=1.55 \mu \mathrm{m}$ with initial pulse width $125 \mathrm{ps}$ for second order dispersion $\beta_{2}=-20 \mathrm{ps}^{2} \mathrm{~km}^{-1}$, nonlinear parameter $\gamma=3 \mathrm{~W}^{-1} \mathrm{~kg}^{-1}$ and initial chirp $C=-0.25$ two near soliton pulses were generated for input powers $P=0.54 \mathrm{~mW}$ and $P=0.64 \mathrm{~mW}$ and a perfect soliton for the hyperbolic secant input pulse.
\end{abstract}

\section{INTRODUCTION}

With the advancement of the information technology, the demand for high bit rate communication systems are increasing. In recent times, there is an extensive research effort to develop and improve all optical fiber based transmission systems. Once the speed of optical fiber systems approaches the theoretical fiber bandwidth, the time duration and intensities of the transmitted optical bits approach strong optical nonlinearities. Therefore to maintain quasi-linear transmission, either pulse shape must undergo compensation or the chromatic dispersion of the different wavelengths in the pulse must be balanced against the fiber nonlinearities exploiting the nonlinear effects. The group velocity dispersion (GVD) imposes severe limit on the information carrying capacity of optical communication systems. By choosing appropriate pulse shape, highly stable light pulses known as solitons which preserve their shape over long distances are generated when the effect of group velocity dispersion (GVD) is balanced by self-phase modulation (SPM). The soliton based optical communication systems can be used over distances of several thousands of kilometers with huge information carrying capacity by using optical amplifiers. When soliton in different channels propagate at different speeds collisions are caused among them. These collisions produce frequency shifts. Although in an ideal collision process due to the symmetric picture of the whole collision process, the frequency shift returns to zero after the collision, when amplifier lump exists in the middle of the collision process, the symmetry breaks down resulting frequency shifts. This frequency shift may be completely cancelled out by dispersion management techniques. The dispersion management techniques are based on finding the solution to nonlinear Schrödinger wave equation. The collision also induces chirp degeneration. The chirped solitons suffer less nonlinear interaction than regular solitons and thereby increase their transmission capacity. In order to realize broadband optical communication systems and networks, it is imperative to compensate the pulse spreading due to group velocity dispersion (GVD) and fiber nonlinearities due to optical Kerr's effects. Different compensation methods like optical phase conjugation method [1] and Bragggrating method [2] have been studied in the last decade mainly to increase the transmission distances and bandwidth of optical communication systems. In techniques known as dispersion management both chromatic dispersion and optical Kerr's effects [3] have been studied together. There is a significant increase in the transmission speed of optical networks when the impact of higher order terms is clarified [4]. A simple technique of pulse compression based on linear chirp 
compensation of self-phase modulation in dispersion-shifted fibers was demonstrated by Calvani et.al [5]. An expression for relative intensity noise due to dispersion and nonlinearity including fiber loss was derived to show its impact with first order dispersion term [6]. The use of SPM and joint optimization of the bias and modulation voltages to increase the dispersion limited transmission distance at $10 \mathrm{~Gb} / \mathrm{s}$ were combined by Cartledge [7]. A general treatment of multi-span effects of Kerr nonlinearity on Shannon channel capacity for dispersion free nonlinear optical fiber transmission has also been studied [8].

In the present work, using Gaussian pulse and hyperbolic secant pulse as input pulses, the split step Fourier method was used to generate soliton pulses inside an optical fiber composed of a glass core surrounded by a cladding layer, varying different parameters such as initial chirp, input power and nonlinear coefficient which can propagates longer distances with lower energy losses by cancelling the effects of GVD and nonlinearity with each other.

\section{INDUCED POLARIZATION}

For intense electromagnetic fields any dielectric medium behaves like a nonlinear medium, the origin of nonlinearity lying in anharmonic motion of the bound electrons. Due to this anharmonic motion the total polarization $P$ induced by electric dipoles satisfies:

$$
P=\sum_{i} \varepsilon_{0} \chi^{i} E^{i}
$$

where $\varepsilon_{0}$ is the permittivity of vacuum and $\chi^{i}(i=1,2, \ldots)$ is $i^{\text {th }}$ order susceptibility. The dominant contribution to $P$ is provided by linear susceptibilit $\chi^{1}$. The second order susceptibility $\chi^{2}$ is responsible for second harmonic generation and sum-frequency generation. A medium, which lacks inversion symmetry at the molecular level, has non-zero second order susceptibility. However for a symmetric molecule like silica, $\chi^{2}$ vanishes. The third order susceptibility $\chi^{3}$, the lowest-order nonlinear effects in fibers, is responsible for Kerr effect. For isotropic medium like optical fibers, as the polarization vector $\boldsymbol{P}$ will always be in direction of electric field vector $\boldsymbol{E}$, the scalar notations instead of vector notations can be used. For an electric field,

$$
E=E_{0} \cos (\omega t-k z)
$$

the polarization $P$ becomes,

$$
P=\varepsilon_{0}\left(\chi^{1} E_{0} \cos (\omega t-k z)+\chi^{2} E_{0}^{2} \cos ^{2}(\omega t-k z)+\chi^{3} E_{0}^{3} \cos ^{3}(\omega t-k z)+\ldots\right)
$$

Using some trigonometric relations, equation 3 can be written as

$$
P=\varepsilon_{0}\left(\chi^{1} E_{0}+\frac{1}{2} \chi^{2} E_{0}{ }^{2}+\frac{3}{4} \chi^{3} E_{0}^{3} \cos (\omega t-k z)+\frac{1}{2} \chi^{2} E_{0}{ }^{2} \cos 2(\omega t-k z)+\frac{1}{4} \chi^{3} E_{0}^{3} \cos 3(\omega t-k z)+\ldots .\right)
$$

As first and second terms of equation 4 are constant terms giving direct current fields across the medium, the effect of them are of little practical importance. The third, fourth and fifth terms oscillating at frequency $\omega$ are known as first or fundamental, second and third harmonic polarizations respectively. Neglecting the third term of the equation 4 due to phase mismatch and the higher order terms because of their negligible contributions, equation 4 reduces to an equation containing both linear and nonlinear polarizations:

$$
P=\varepsilon_{0}\left(\chi^{1} E_{0}+\frac{3}{4} \chi^{3} E_{0}^{3} \cos (\omega t-k z)\right)
$$


For a plane wave represented in equation 2, the intensity $I$ is defined as,

$$
I=\frac{1}{2} \varepsilon_{0} c n_{l} E_{0}^{2}
$$

where $\mathrm{c}$ is velocity of light and $n_{l}$ is linear refractive index of the medium at low fields. Hence polarization in terms of intensity read:

$$
P=\varepsilon_{0} E_{0}\left(\chi^{1}+\frac{3}{2}\left(\frac{\chi^{3}}{\varepsilon_{0} c n_{l}}\right) I \cos (\omega t-k z)\right)
$$

The effective susceptibility $\chi_{e f f}$ of the medium is defined as,

$$
\chi_{\text {eff }}=\frac{P}{\varepsilon_{0} E_{0}}=\chi^{1}+\frac{3}{2}\left(\frac{\chi^{3}}{\varepsilon_{0} c n_{l}}\right) I
$$

Therefore, effective refractive index $n_{\text {eff }}$ :

$$
n_{e f f}=\left(1+\chi_{e f f}\right)^{1 / 2}=\left(1+\chi^{1}+\frac{3}{2}\left(\frac{\chi^{3}}{\varepsilon_{0} c n_{l}}\right) I\right)^{1 / 2}
$$

The last term is usually very small even for very intense light beam. Hence the expression in equation 9 can be expanded using Taylor series expansion:

$$
n_{\text {eff }}=n_{l}+\frac{3}{4}\left(\frac{\chi^{3}}{\varepsilon_{0} c n_{l}}\right) I=n_{l}+n_{n l} I
$$

where $n_{l}$ and $n_{n l}$ refer to linear and nonlinear refractive indices with $n_{l}=\left(1+\chi^{3}\right)^{1 / 2}$. Although, this change in refractive index is very small, due to the very long interaction length $(10-10,000 \mathrm{~km})$ of an optical fiber, the accumulated effects become significant and the nonlinear term is that is responsible for the formation of a soliton. For longer fiber link lengths as more light interacts, the nonlinear effect become greater. Due to fiber attenuation, the power of the propagating optical beam decreases along the length of the fibre and the optical power at a distance $z$ along the link is

$$
P(z)=P_{i n} e^{-\alpha z}
$$

where $P_{i n}$ is the input power at $\mathrm{z}=0$ and $\alpha$ is the coefficient of attenuation. The effective length $L_{\text {eff }}$, the length up to which the power is assumed to be constant is defined for an actual link length $L$ as:

$$
P_{i n} L_{e f f}=\int_{0}^{L} P(z) d z
$$

From equations 11 and 12, the effective link length can be obtained as,

$$
L_{e f f}=\frac{1-e^{-\alpha z}}{\alpha} \text {. }
$$

Since for communication fibers, the actual length $L \gg 1 / \alpha$, the effective link length is $L_{\text {eff }} \simeq 1 / \alpha$. 
The effect of nonlinearity grows with the increase in intensity of the beam in the fiber. The intensity is inversely proportional to area of the core. Effective cross-sectional area $A_{\text {eff }}$ is related to the actual area $A$ and the cross-sectional distribution of intensity $I(r, \theta)$ in plane polar coordinates can be written in the following way:

$$
A_{e f f}=\frac{\iint_{r} r d r d \theta I(r, \theta)}{\iint_{r} r d r d \theta I^{2}(r, \theta)}
$$

\section{NON-LINEAR EFFECTS}

Nonlinear effects in optical fibers occur due to change in the refractive index of the medium with optical intensity and inelastic-scattering phenomenon. At high power level, the inelastic scattering phenomenon can induce stimulated Brillouin-scattering and Raman-scattering. When the electric field is due to the light itself, the variation in refraction index, proportional to the local irradiance of the light leads to Kerr effect. This is responsible for the nonlinear optical effects and become significant with very intense beams such as laser beams. Depending upon the type of input signal, the Kerr-nonlinearity manifests itself in three different effects such as self-phase modulation, crossphase modulation and four-wave mixing. An ultra-short pulse light, when travelling in a medium, will induce a varying refractive index due to the optical Kerr effect producing a phase shift in the pulse, leading to a change of the pulse's frequency spectrum known as self-phase modulation (SPM). The primary effect of SPM is to broaden the spectrum of the pulse, keeping the temporal shape unaltered.

In digital communication systems, the information encoded in the form of pulses, in the process of transmitting from the transmitter to the receiver along the fiber, spread out creating pulse dispersion. A typical fiber optic communication system using a pulse wavelength of $1.55 \mu \mathrm{m}$, falls within the anomalous dispersion regime. The group velocity dispersion is the group delay dispersion per unit length:

$$
G V D=\frac{\partial}{\partial \omega}\left(\frac{1}{v_{g}}\right)=\frac{\partial^{2} k}{\partial \omega^{2}}
$$

where $v_{g}$ is the group velocity of the pulse and $k$ is the frequency-dependent wave number.

The simplest model for pulse propagation in optical fiber including nonlinear effects is the generalized nonlinear Schrodinger equation (NLSE) [9]:

$$
\frac{\partial A}{\partial z}=-i \frac{\beta_{2}}{2} \frac{\partial^{2} A}{\partial t^{2}}+\frac{\beta_{3}}{6} \frac{\partial^{3} A}{\partial t^{3}}-\frac{\alpha}{2} A+i \gamma|A|^{2} A+\Lambda \frac{\partial}{\partial t}\left(|A|^{2}\right) A
$$

where $A(z, t)$ is the amplitude modulation function of the rapidly oscillating electric field in a coordinate system moving with the signal, $\beta_{2}$ is the group velocity dispersion (GVD), $\beta_{3}$ is the third order dispersion (TOD), $\alpha$ is the attenuation coefficient, $\gamma$ is the self-phase modulation (SPM) coefficient and $\Lambda$ is the self-steepening. For soliton generation that cancels the effects of group velocity dispersion from nonlinearity effects, the third order dispersion term $\beta_{3}$ becomes negligible for picoseconds pulses. Therefore equation 16 reduces to:

$$
\frac{\partial A}{\partial z}=-i \frac{\beta_{2}}{2} \frac{\partial^{2} A}{\partial t^{2}}-\frac{\alpha}{2} A+i \gamma A|A|^{2}
$$


where the self-phase modulation coefficient $\gamma=2 \pi n_{2} / \lambda A_{\text {eff }}$ and the group velocity dispersion parameter $\beta_{2}=\partial^{2} \beta / \partial \omega^{2}$ is given in terms of is the phase constant $\beta$ of each frequency. $\omega$ is the frequency of the pulse and $\lambda$ is the optical wavelength.

The dispersion length $L_{D}$ is the length up to which the effects of chromatic dispersion or group velocity dispersion are negligible while the nonlinear length $L_{N L}$ represents the length, up to which the effects of nonlinearity or self-phase modulation are negligible. If the length of the medium is very much greater than the dispersion length $L \gg L_{D}$ and nonlinear length $L \gg L_{N L}$, then pulse undergo both the effect of group velocity dispersion and self-phase modulation and a soliton can be generated.

\section{SOLITON}

A soliton is a self-reinforcing solitary wave packet or pulse that maintains its shape while it travels at constant speed. If the pulse has just the right shape, the Kerr effect will exactly cancel the dispersion effect, and the pulse's shape will not change over the time creating a soliton. Using normalization parameters such as normalized time $\tau=T / T_{0}$, normalized distance $\xi=z / L_{D}$ and the normalized pulse envelope function $u=A / \sqrt{P_{0}}$, and neglecting the energy loss to the fiber as $\alpha$ is very small, the nonlinear Schrödinger equation can be written as

$$
\frac{\partial u}{\partial \xi}-i \frac{s}{2} \frac{\partial^{2} u}{\partial \tau^{2}}+i N^{2}|u|^{2} u=0
$$

where $s=-1$ for $\beta_{2}<0$ (anomalous GVD-Bright Soliton) and $N$ is an integer designating the order of soliton given by

$$
N=\frac{L_{D}}{L_{N l}}=\frac{\gamma P_{0} T_{0}^{2}}{\left|\beta_{2}\right|}
$$

SPM dominates for $N>1$ while dispersion effects dominates for $N<1$. For $N \approx 1$ both SPM and GVD cooperate in such a way that the SPM-induced chirp is just right to cancel the GVD-induced broadening of the pulse. The optical pulse would then propagate undistorted in the form of a soliton. By integrating the NLSE, the solution for fundamental soliton $(N=1)$ can be written as,

$$
u(\xi, \tau)=\sec h(\tau) e^{i \xi / 2}
$$

\footnotetext{
Since dispersion and nonlinear effects act simultaneously on propagating pulses in optical fibers, the NLSE cannot be analytically solved. Hence the numerical split step Fourier method was utilized, by breaking the entire

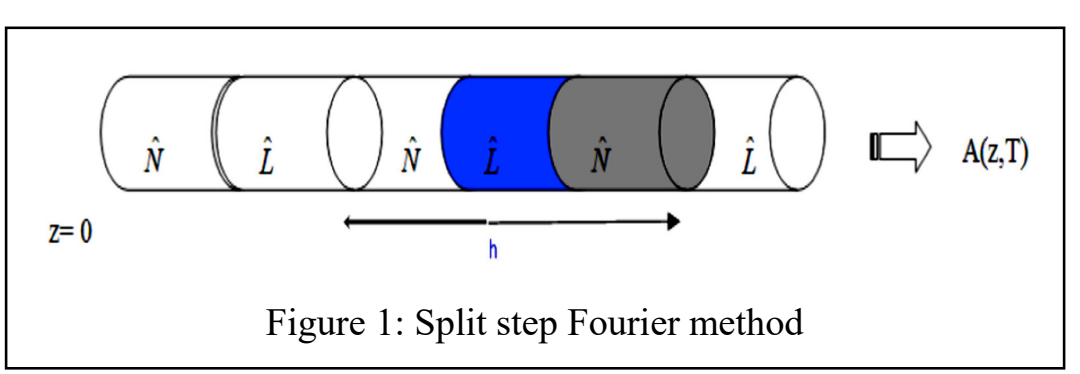
length of the fiber into small step sizes of length, $h$ and then splitting it into two halves, linear part (dispersive part) and nonlinear part over $z$ to $z+h$ (Figure 1), the nonlinear Schrödinger equation was solved. Each part was solved individually and then combined together afterwards to obtain the aggregate output of the traversed pulse. The linear dispersive part was solved first in the Fourier domain using the fast Fourier transforms and then inverse Fourier transformed into the time domain
} 
to solve the equation for the nonlinear term before combining them. The process is repeated over the entire span of the fiber, to approximate the nonlinear pulse propagation. The value of $h$ is chosen for the peak power $P_{0}(z, t)$ with maximum phase shift $\phi_{\max }=\gamma\left|P_{0}\right|^{2} h=0.05 \mathrm{rad}$. The solution of the generalized Schrödinger equation is described using this method [10]:

$$
\frac{\partial A}{\partial z}=\left(-i \frac{\beta_{2}}{2} \frac{\partial^{2} A}{\partial T^{2}}+\frac{\beta_{3}}{6} \frac{\partial^{3} A}{\partial T^{3}}-\frac{\alpha}{2} A\right)+i \gamma\left(|A|^{2}+\frac{i}{\omega_{0} A} \frac{\partial\left(|A|^{2} A\right)}{\partial T}-T_{R} \frac{\partial|A|^{2}}{\partial T}\right) A=(\hat{L}+\hat{N}) A
$$

where a frame of reference moving with the pulse at the group velocity is used by making the transformation $T=t-\beta_{1} z$ and $T_{R}$ is the first moment of the nonlinear response function. For a pulse of width $T_{0}>5 \mathrm{ps}$ the last two terms can be neglected and the third order dispersion term is also quite small. The linear part $\hat{L}$ and nonlinear part $\hat{N}$ are separated. The split step Fourier method was implemented for Gaussian pulse and hyperbolic secant pulse:

$$
A(z+h, T) \approx A(z, T) e^{h \hat{L}} e^{h \hat{N}}
$$

The exponential operator $e^{h \hat{L}}$ can be evaluated in the Fourier domain. By taking pulse width, $125 \mathrm{ps}$ we have assume $\alpha=0, \beta_{3}=0$ and higher order nonlinear effects of self-steepening to be zero.

\section{GAUSSIAN PULSE}

A Gaussian pulse with initial chirp $C$, input amplitude $A_{0}$ and pulse width $T_{0}$ is given by [11]:

$$
u(0, t)=A_{0}^{*} e^{-(1+i C) t^{2} / 2 T_{0}^{2}}
$$

The spectral amplitude of the pulse is given by the Fourier transform $\operatorname{FT}(u(L, 0))$ :

$$
U(L, \omega)=\mathrm{FT}(u(L, t))=\int_{-\infty}^{+\infty} u(L, t) e^{i \omega t} d t
$$

In the presence of group velocity dispersion, the different frequency components undergo a phase change. The rate of phase change respect to time due to GVD is:

$$
\delta \omega=\frac{d \phi}{d t}=\frac{\left(z / L_{D}\right) \sin \beta_{2}}{1+\left(z / L_{D}\right)^{2}} \frac{t}{T_{0}^{2}}
$$

The frequency chirp due to self-phase modulation can be shown as,

$$
\omega^{\prime}=\omega_{0}-\frac{2 \pi}{\lambda} L_{e f f} n_{n l} \frac{d I}{d t}=\omega_{0} \mp \omega(t) .
$$

Minus sign represents the leading edge of the pulse and the positive sign the trailing edge of the pulse. This shows that the pulse is chirped. This chirping phenomenon is generated due to SPM, which leads to the spectral broadening of the pulse. 
For a Gaussian pulse, the dispersion length $L_{D}$ and nonlinear length $L_{n l}$ is given by:

$$
L_{D}=\frac{T_{0}^{2}}{\left|\beta_{2}\right|}, \quad L_{n l}=\frac{1}{\gamma^{P}}
$$

where $T_{0}$ is the pulse width and $P$ is the power of the pulse. The simplified nonlinear Schrodinger equation:

$$
\frac{\partial A}{\partial z}=\left(-i \frac{\beta_{2}}{2} \frac{\partial^{2} A}{\partial t^{2}}-\frac{\alpha}{2} A\right)+\left(i \gamma A|A|^{2}\right)=(\hat{L}+\hat{N}) A
$$

was solved in two dispersion steps with a nonlinear step in the middle. This process was repeated over the length of the fiber. A Gaussian pulse at a wavelength of $\lambda=1.55 \mu \mathrm{m}$ with an input power $P=0.64 \mathrm{~mW}$, initial pulse width $T_{0}=125 \mathrm{ps}$ and initial chirp $C=0.5$ was considered as the input pulse. This is shown in figure 1 for normalized time. A standard telecommunication fiber with nonlinear parameter $\gamma=3 \mathrm{~W}^{-1} \mathrm{~km}^{-1}$, second order dispersion and $\beta_{2}=-20 \mathrm{ps}^{2} \mathrm{~km}^{-1}$ was used in Matlab simulation. The dispersion length was $L_{D}=781.25 \mathrm{~m}$.

At the half of the maximum amplitude of the pulse, the width of the pulse is called full-width half-maximum (FWHM) and this is shown in figure 2. The pulse broadening was calculated as the ratio of FWHM of the propagating pulse to FWHM of the input pulse. To generate a soliton, this ratio must be one throughout all steps of propagation.

For nonlinear parameter $\gamma=3 \mathrm{~W}^{-1} \mathrm{~km}^{-1}$, and input power $P=0.64 \mathrm{~mW}$, the pulse

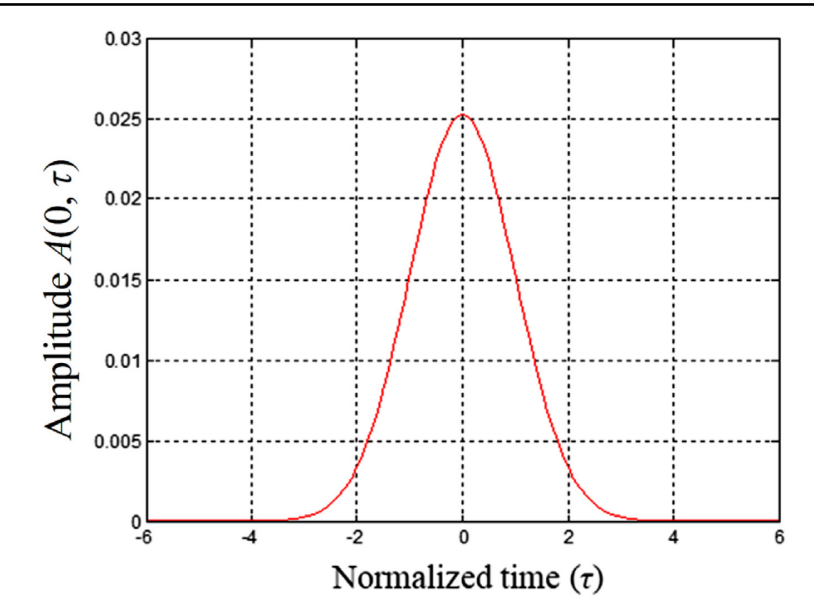

Figure 1: Input Gaussian pulse

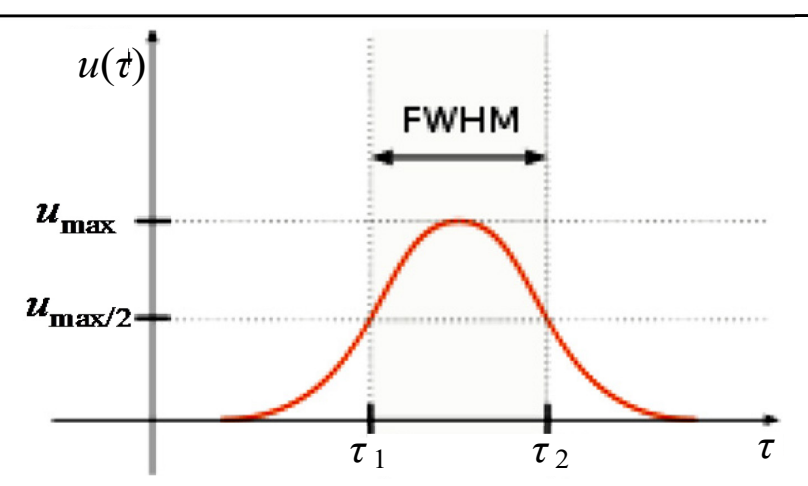

Figure 2: Full width at half maximum broadening ratio for various initial chirps, $C=-0.5,-0.25,0.0,0.25,0.5$ are shown in figure 3 with GVD and SPM acting simultaneously on the Gaussian pulse. For the initial chirp of -0.5 , the evolution pattern given by the blue line shows that pulse broadens at first for a small period of length. But gradually the rate at which it broadens slowly declines and the pulse broadening ratio reach a constant value. The pulse moves at a slightly larger but constant width as it propagates along the length of the fiber. For an initial chirp of -0.25 shown by red line, the pulse starts to broaden at first but after a small period of length starts to narrow at decreasing rate and reaches a constant value. If both GVD and SPM act simultaneously on the propagating Gaussian pulse with no initial chirp $(C=0)$, shown by black line, the pulse shrinks initially for a very small period of propagating length. After that the broadening ratio reaches a constant value and a stable pulse is propagated. When the initial chirp is positive, pulse tends to shrink rapidly in first period of length but the rate at which it narrows slowly decreases and then tries to gain the original pulse width after reaching a minimum pulse width. The GVD and SPM effects may cancel each other out when the 
GVD induced negative chirp equals the SPM induced positive chirp. But in this case, the initial chirp affects the both GVD and SPM behaviours. When the chirp parameter has a negative value, it is added to the negative chirp of the GVD and subtracted from the positive chirp of SPM causing the net value of chirp to be negative. This means that GVD is dominant during the early stages of propagation causing broadening of the pulse. When the chirp is positive, it is added to the positive chirp of the SPM and subtracted from the negative chirp of GVD causing the net value of chirp to be positive. That is the SPM is

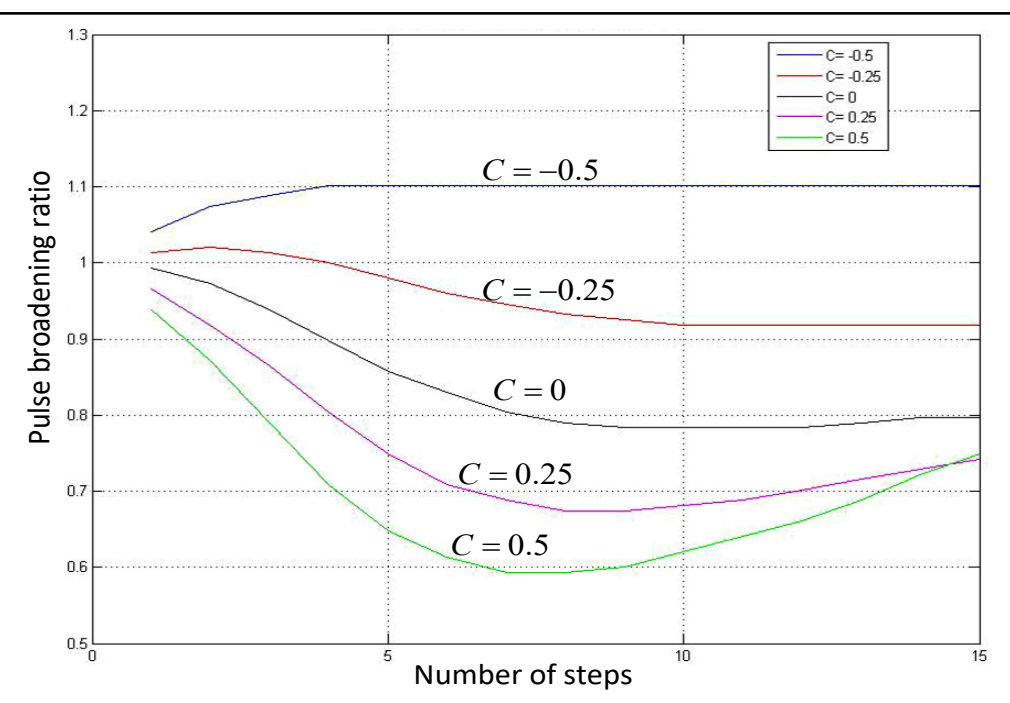

Figure 3: Pulse broadening ratio for input power $P=0.64 \mathrm{~mW}$, $\gamma=3 \mathrm{~W}^{-1} \mathrm{~km}^{-1}$ and initial chirps $C=-0.5,-0.25,0.0,0.25,0.5$. dominant during the early stages of propagation causing narrowing of the pulse. But as the propagation distance increases the effect of the initial chirp decreases while the induced chirp effects of both GVD and SPM regains control. The difference between positive and negative induced effect is lessened and the GVD and SPM effects eventually cancel out each other to propagate at constant width.

Pulse broadening ratio for various input powers $(0.44,0.54,0.64,0.74,0.84) \mathrm{mW}$ for nonlinear parameter $\gamma=3 \mathrm{~W}^{-1} \mathrm{~km}^{-1}$ and initial chirp of $C=0$ is shown in figure 4 . The pulse broadening ratio of a pulse with a smaller input power is higher than for those with larger input power as the nonlinear length is inversely proportional to the input power. As the length of the medium (fiber) is much longer than the dispersion length $\left(L_{D}\right)$ and the nonlinear length $\left(L_{n l}\right)$, the pulse undergoes both the GVD and SPM effects. A pulse undergoes nonlinear effects (SPM) much earlier when $L_{n l}$ is shorter as the pulse needs to propagate only a lesser length of the medium to be affected by this effect. Therefore when input power of the pulse increases, the nonlinear length is decreased so the pulse undergoes

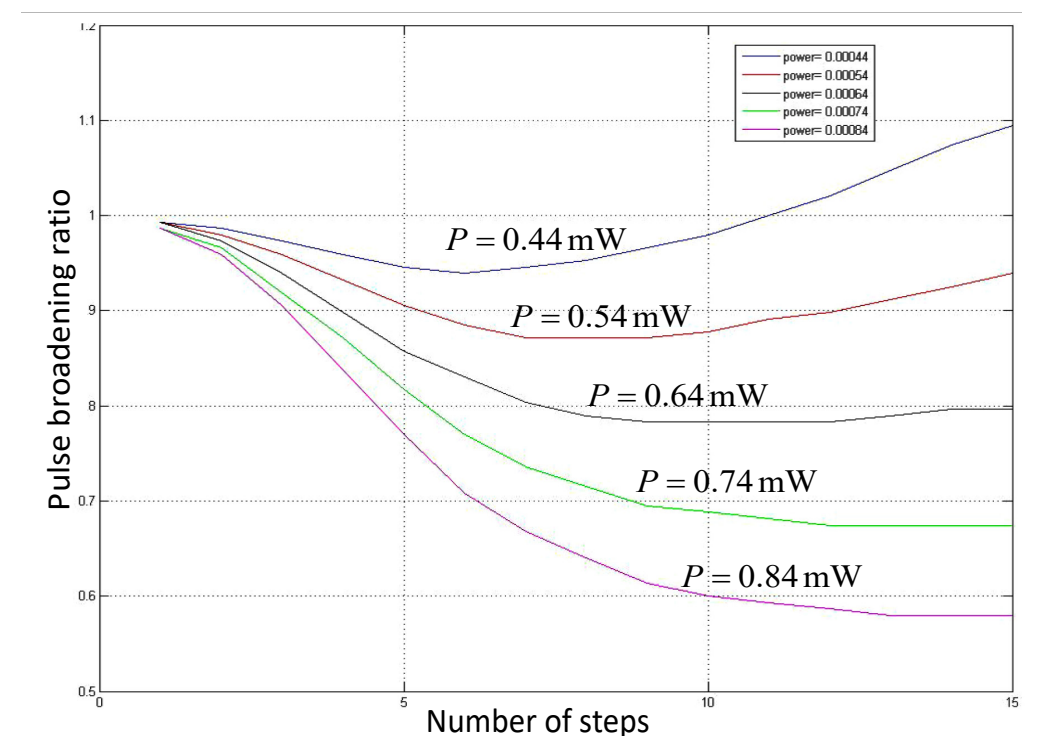

Figure 4: Pulse broadening ratio for $\gamma=3 \mathrm{~W}^{-1} \mathrm{~km}^{-1}, C=0$ and input powers $(0.44,0.54,0.64,0.74,0.84) \mathrm{mW}$

SPM much earlier and at the beginning, the pulse starts to shrink when the input power of the pulse increases. 
The pulse broadening ratio for various nonlinear parameter $\gamma=(1,2,3,4,5) \mathrm{W}^{-1} \mathrm{~km}^{-1}$ for initial chirp $C=0$ and input power is shown in figure 5 . When the value of the nonlinear parameter $\gamma$ increases, the nonlinear length decreases so that the pulse undergoes SPM much earlier. Therefore we can see from the figure 5 as $\gamma$ increases, the SPM dominates and the pulse shrinks. But as the pulse starts to propagate through the medium, it feels the effects of GVD more and negative chirp of GVD starts to cancel out

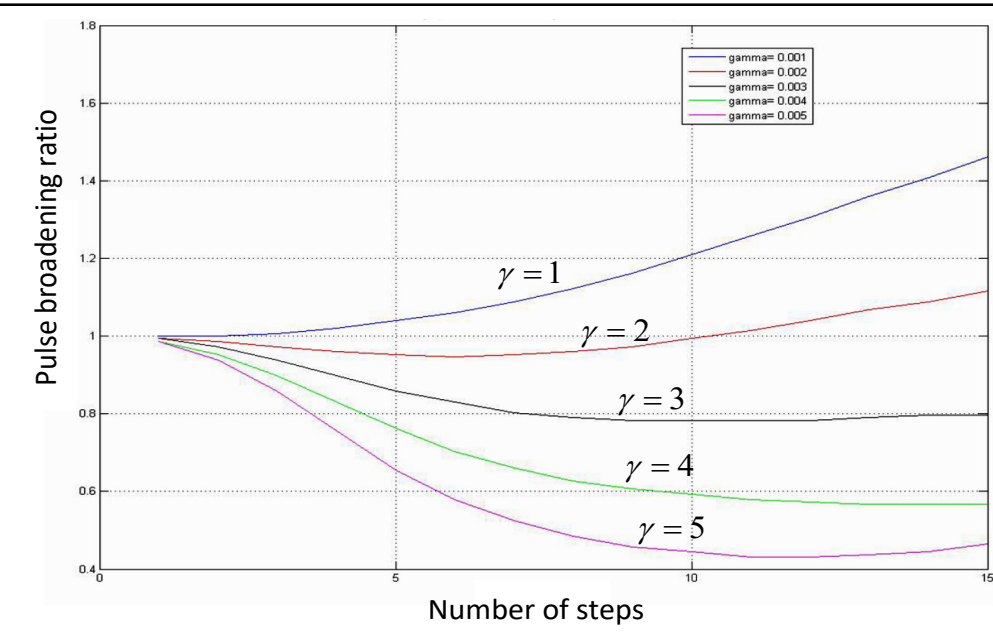

Figure 5: Pulse broadening ratio for input power $P=0.64 \mathrm{~mW}$,

$$
C=0 \text { and } \gamma=(1,2,3,4,5) \mathrm{W}^{-1} \mathrm{~km}^{-1}
$$
the positive chirp of the SPM decreasing the pulse narrowing rate. Then it starts to propagate with a constant pulse width, if both SPM and GVD cancel out each other's effects or pulse width is increased so that GVD is dominant. But when $\gamma=1 \mathrm{~W}^{-1} \mathrm{~km}^{-1}$, the negative chirp of the GVD is much larger than the positive chirp of the SPM therefore from the beginning GVD dominates so that the pulse start broadening from the beginning.

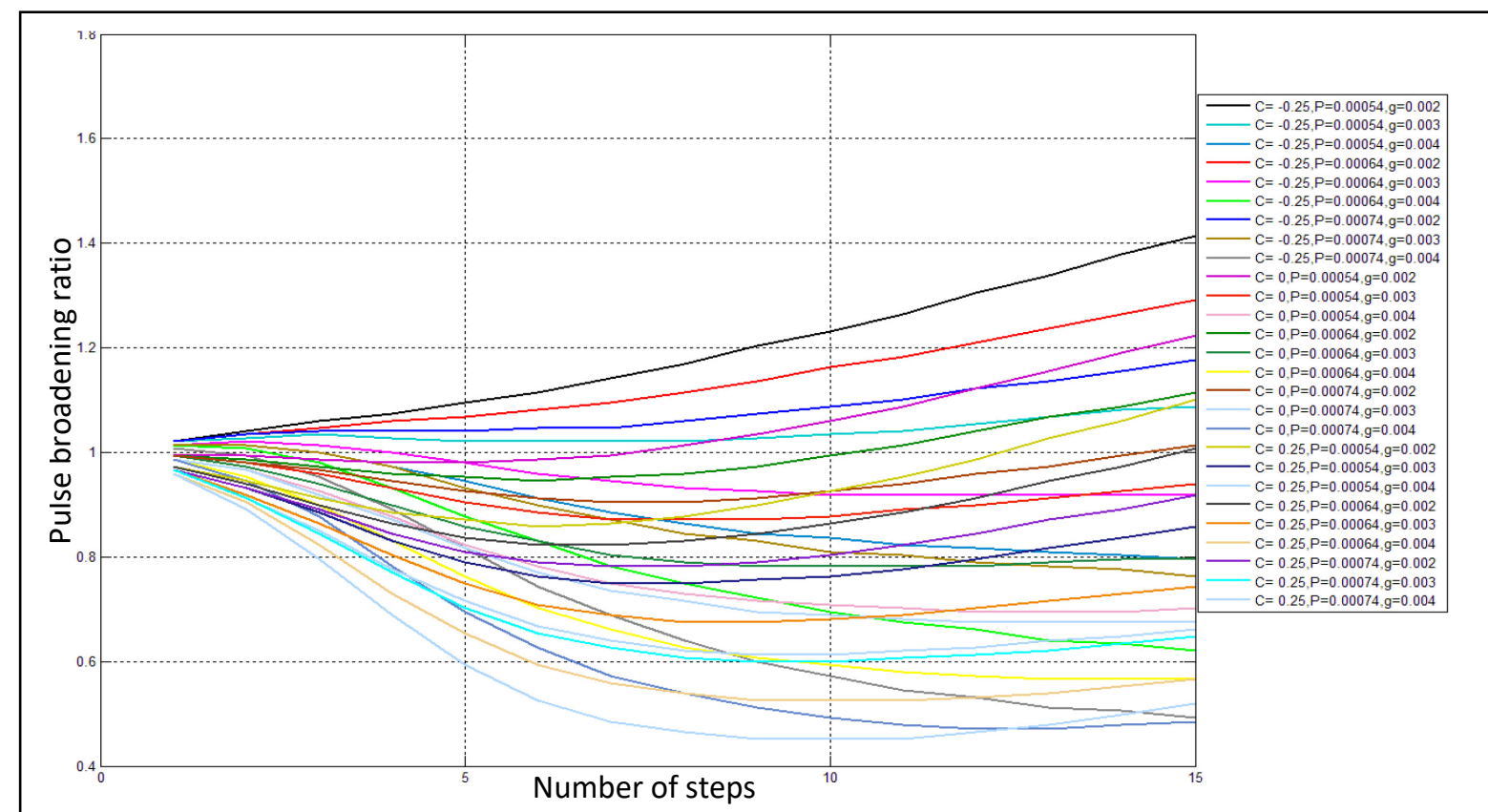

Figure 6: Pulse broadening ratio for input powers $P=(0.54,0.64,0.74) \mathrm{mW}$, initial chirps

$$
C=-0.25,0.0,0.25 \text { and } \gamma=(2,3,4) \mathrm{W}^{-1} \mathrm{~km}^{-1} \text {. }
$$

Pulse broadening ratio for input powers $(0.54,0.64,0.74) \mathrm{mW}$, initial chirps $C=-0.25,0.0,0.25$ and $\gamma=(2,3,4) \mathrm{W}^{-1} \mathrm{~km}^{-1}$ were obtained to find the optimum values for pulse broadening ratio close to one. This is shown in figure 6 . The figure shows that when $C=0$, $P=0.74 \mathrm{~mW}$ at $\gamma=2 \mathrm{~W}^{-1} \mathrm{~km}^{-1}$ and $C=0.25, P=0.64 \mathrm{~mW}$ at $\gamma=2 \mathrm{~W}^{-1} \mathrm{~km}^{-1}$, the end pulses reach to its original pulse widths although it experience some decrease in the middle. But in both cases in the end we can see the pulse is broadening its way to original width and if the "number of steps' had been increased the pulse would have been broaden more than its original width and the 
pulse broadening ratio would have been more than one. Therefore these cases cannot be chosen as the optimum values for this setup. When $C=-0.25, \quad P=0.54 \mathrm{~mW} \quad$ at $\gamma=3 \mathrm{~W}^{-1} \mathrm{~km}^{-1}$ the propagation pulse broadening ratio is almost one and always stays at the upper side of the margin one of the pulse broadening ratio. At the end of the propagation it gains a constant pulse width so the pulse broadening ratio becomes constant which is less than 1 . When

$C=-0.25, P=0.64 \mathrm{~mW}, \gamma=3 \mathrm{~W}^{-1} \mathrm{~km}^{-1}$

the propagation pulse broadening ratio is almost one and always stays at the lower side of the margin one of the pulse broadening ratio. At the end of the propagation it gains a constant pulse width so the pulse broadening ratio becomes constant which is more than 0.9 . Therefore we could choose both the above cases as the optimum values for this setup as the pulse width become constant at the end in both cases and both pulses have almost their original pulse width at the end. Pulse evolutions of these two cases are shown in figure 7. When the input power is $P=0.54 \mathrm{~mW}$, the amplitude of the output pulse is slightly smaller $(\simeq 0.023)$ than the input pulse magnitude 0.025 with a pulse broadening ratio greater than one. When the input power is $P=0.64 \mathrm{~mW}$, the amplitude of the output pulse is slightly larger $(\simeq 0.026)$ than the amplitude of the input pulse, 0.025 as the pulse is narrower

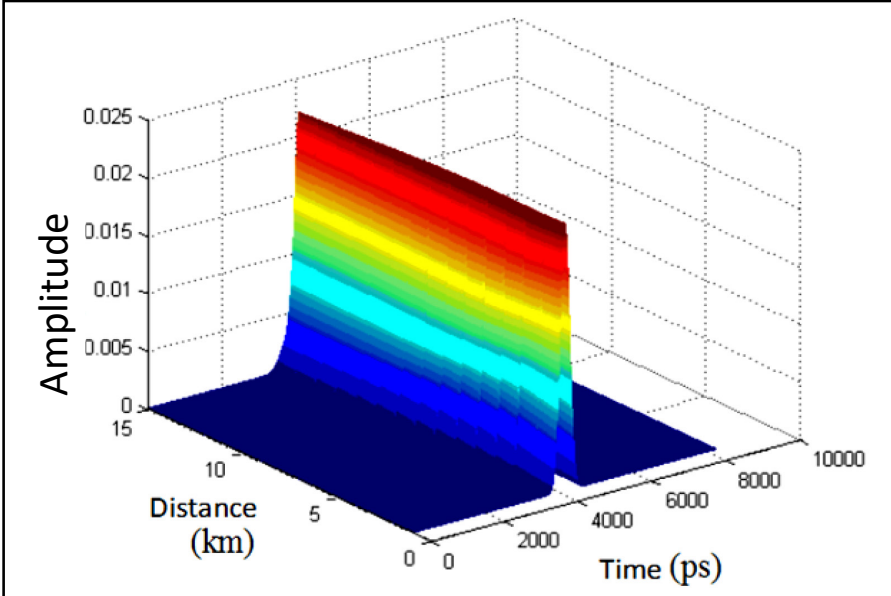

Figure 7(a): Pulse evolution at $C=-0.25$, $\gamma=3 \mathrm{~W}^{-1} \mathrm{~km}^{-1}, P=0.54 \mathrm{~mW}, A=0.023$

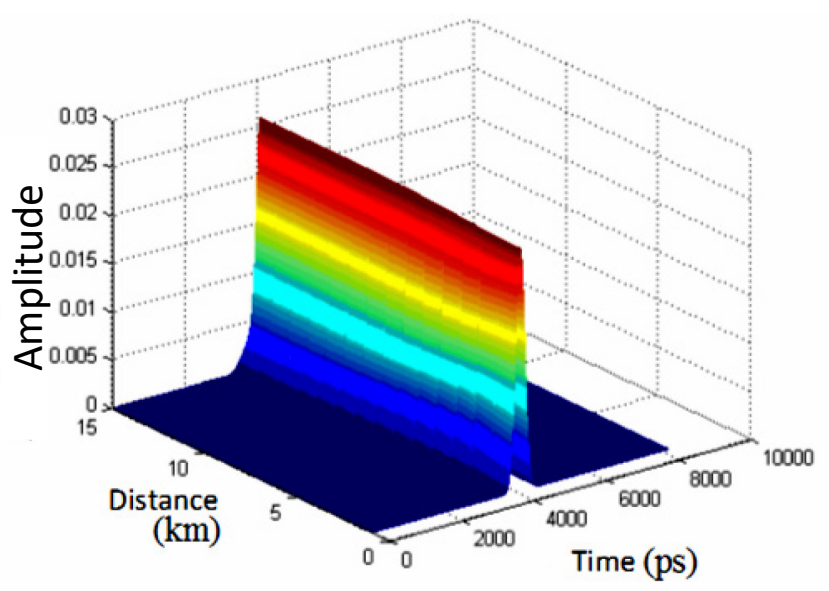

Figure 7(b): Pulse evolution at $C=-0.25$, $\gamma=3 \mathrm{~W}^{-1} \mathrm{~km}^{-1}, P=0.64 \mathrm{~mW}, A=0.025$ with a pulse broadening ratio less than one.

\section{HYPERBOLIC SECANT PULSE}

The hyperbolic-secant pulse shape that occurs naturally in the context of optical solitons and pulses emitted from some mode-locked lasers is of particular interest. For $N \approx 1$ both SPM and GVD cooperate in such a way that the SPM-induced chirp is just right to cancel the GVD-induced broadening of the pulse. The optical pulse would then propagate undistorted in the form of a soliton. By integrating the nonlinear Schrödinger equation, given in equation 18 for fundamental soliton with $N=1$, the following general solution can be obtained for the optical field:

$u(\xi, \tau)=\operatorname{sech}(\tau) e^{i \xi / 2}, \quad u(0, \tau)=\operatorname{sech}(\tau) e^{-i C \tau^{2} / 2}$ 
where $\operatorname{sech}(\tau)=\operatorname{sech}\left(t / T_{0}\right)$ is hyperbolic secant function. Since the phase term $\exp (i \xi / 2)$ has no influence on the shape of the pulse, the soliton is independent of $\xi$ and hence is non-dispersive in time domain. It is this property of a fundamental soliton that makes it an ideal candidate for optical communications. Optical solitons are very stable against perturbations and can be created even when the pulse shape and peak power deviates from the ideal conditions corresponding to $N=1$. A hyperbolic secant pulse

$$
u(0, \tau)=N \operatorname{sech}(\tau) \quad(N=1)
$$

of input power $P=0.64 \mathrm{~mW}$ and initial pulse width $125 \mathrm{ps}$ was considered. This pulse with normalized time is shown in figure 8. A standard telecommunication fiber with nonlinear parameter $\gamma=3 \mathrm{~W}^{-1} \mathrm{~km}^{-1}$, second order dispersion and $\beta_{2}=-20 \mathrm{ps}^{2} \mathrm{~km}^{-1}$ was used in Matlab simulation. The dispersion length was $L_{D}=520.8 \mathrm{~m}$. In the general case, the spectrum of the transmitted pulse cannot be calculated analytically and only an approximate expression giving the bandwidth of the transmitted spectrum is used. The spectral amplitude of the pulse at the output of a fiber of length $L$ is given by the Fourier transforms in equation 24. The intensity spectrum of the transmitted pulse $|U(L, \omega)|^{2}$, the self-phase modulation spectrum is symmetrical and that its effect is independent of the sign of the nonlinear coefficient when dispersion is neglected. Hyperbolic secant pulse propagation without initial chirp was simulated by analytically solving the normalized Schrodinger equation given in equation 18 with $s=-1$ and $N=1$ using the split step

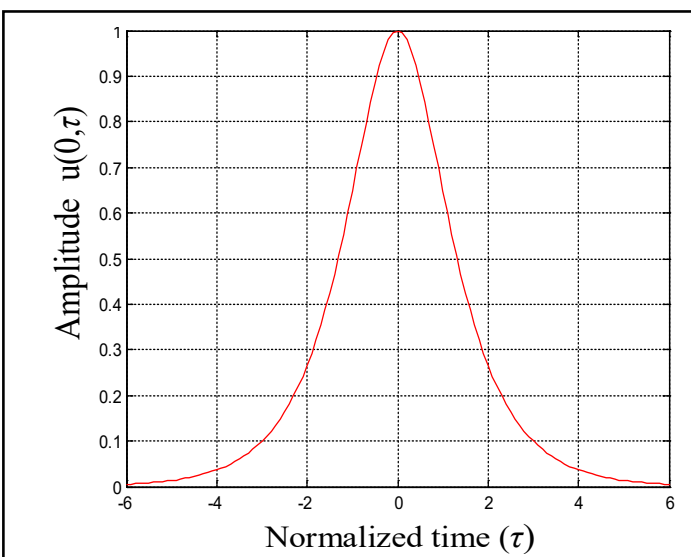

Figure 8: Input hyperbolic secant pulse with amplitude

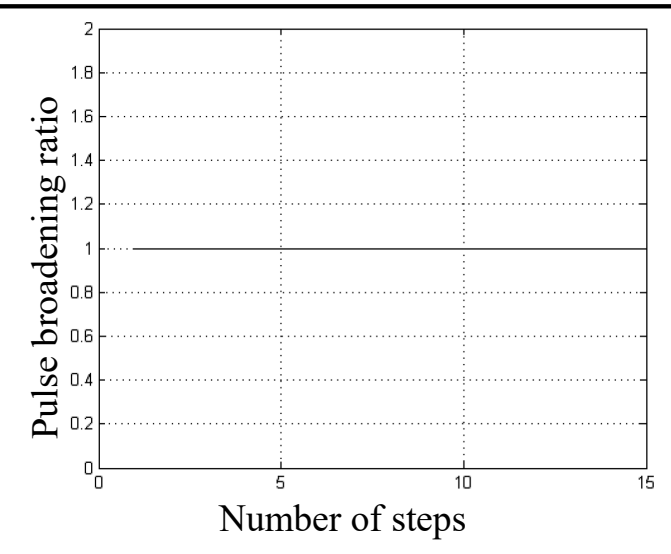

Figure 9: Pulse broadening ratio for hyperbolic secant pulse

Fourier method. The pulse broadening ratio obtained for an input pulse of power $P=0.64 \mathrm{~mW}$ with normalized amplitude and nonlinear parameter $\gamma=3 \mathrm{~W}^{-1} \mathrm{~km}^{-1}$ is shown in figure 9. The steady, horizontal line shows that the pulse propagates at seemingly constant input width. The propagation of a constant width hyperbolic secant pulse means that for a nonlinear optical fiber, soliton propagation has been achieved. We assumed that no attenuation was present for simplification of solution. The GVD and the SPM manifest themselves through their induced

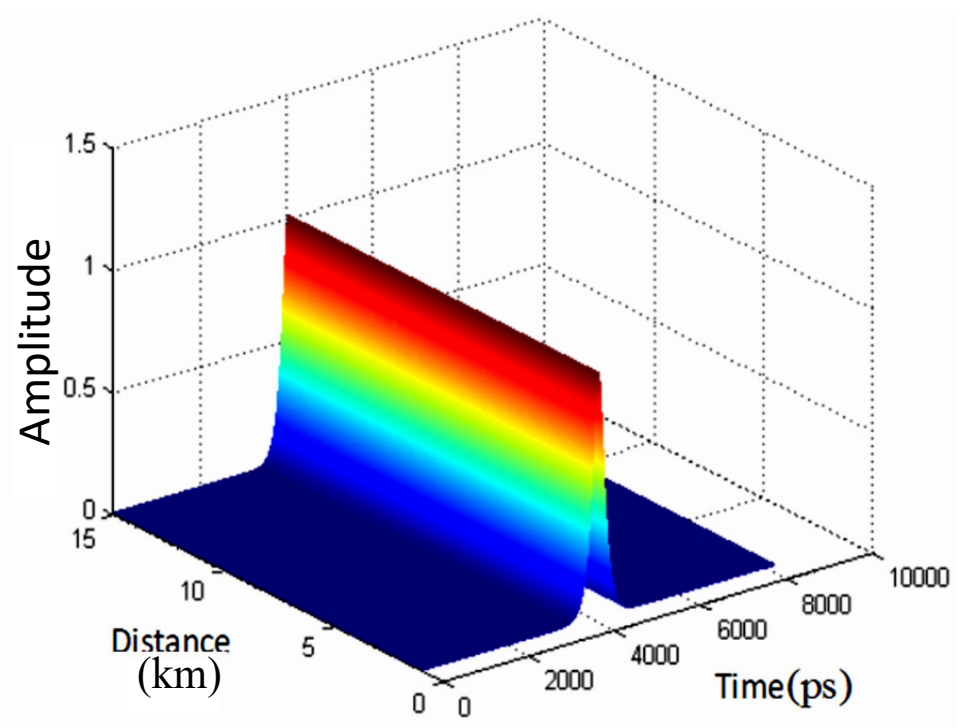

Figure 10: Pulse evolution of a hyperbolic secant pulse of $P=0.64 \mathrm{~mW}$ 
chirp effects. For GVD, the high frequency components of the pulse travel at higher velocity than the low frequencies which induces negative chirp causing dispersion. SPM on the other hand, induces positive chirp during propagation causing the pulse to narrow as it evolves. The net outcome is that the negative induced chirp of GVD cancels out the positive induced chirp of SPM equally producing a fundamental soliton $(N=1)$. The pulse evolution is shown in figure 10 .

\section{CONCLUSION}

When the speed of optical fiber systems approaches the theoretical fiber bandwidth, the time duration and intensities of the transmitted optical bits approaches strong optical nonlinearities. Therefore the nonlinear effects were utilized to balance chromatic dispersion of the different wavelengths in the pulse against the fiber nonlinearities through soliton generation. A Gaussian pulse is affected by both dispersive and nonlinear effects when propagating through an optical fiber. Therefore by varying these parameters for a standard telecommunication fiber composed of a glass core surrounded by a cladding layer at wavelength $\lambda=1.55 \mu \mathrm{m}, \beta_{2}=-20 \mathrm{ps}^{2} \mathrm{~km}^{-1}$ for a Gaussian pulse of input powers of $P=0.54 \mathrm{~mW}$ and $P=0.64 \mathrm{~mW}$ with pulse width $T_{0}=125 \mathrm{ps}$ for the optimum values of the initial chirp $C=-0.25$, nonlinear coefficient $\gamma=3 \times 10^{-3} \mathrm{Wm}^{-1}$ two solitons were generated when the attenuation coefficient of the optical fiber is very small. For a hyperbolic secant input pulse of input power $P=0.64 \mathrm{~mW}$ with pulse width $T_{0}=125 \mathrm{ps}$, throughout the propagation of the pulse it maintained its original pulse shape without being affected by GVD and SPM acting as a perfect soliton by cancelling out negative chirp of the GVD by positive chirp of the SPM. Therefore hyperbolic secant pulse can be used for optical fiber communication systems which has long distance and very small attenuation.

\section{REFERENCES}

[1] G. P.Agrawal, "Soliton Systems" in Fiber-Optic Communication Systems, Ed. New York: Wiley, pp. 404-469, 2002.

[2] K. O. Hill and G. Meltz, "Fiber Bragg Grating Technology Fundamentals and Overview" J. Lightw. Technol. vol.15, no. 8, pp. 1267-1275, 1997.

[3] J. Kerr, "On rotation of the plane of polarization by reflection from the pole of a magnet", Phil. Mag. vol.3, p.321, 1877, "On reflection of polarized light from the equatorial surface of a magnet", Phil. Mag. vol.5, p.161, 1878.

[4] M. Amemiya, "Pulse broadening due to higher order dispersion and its transmission limit", $J$. Lightw. Technol., vol. 20, no. 4, pp. 591-597,. Apr. 2002.

[5] R. Calvani, R. Caponi, E. Grazioli, "Fem to second Transform- limited pulse generation by compensating for linear chirp of SPM spectra in Dispersion Shifted Fibers", J. Fiber and integrated Optics, vol.17, no.1, pp.41-50,1998.

[6] A.V.T Cartaxo, B. Wedding, W. Idler, "Influence of Fiber Nonlinearity on the Fiber Transfer Function: Theoretical and Experimental Analysis", J. Lightw. Technol. vol.17, no.10, pp.1806-, 1999.

[7] J.C. Cartledge, "Combining SPM and Optimum Conditions to Improve the Performance of 10 Gb/s Transmission Systems Using MQW Mach-Zehnder Modulators", J. Lightw.Technol., vol.18, no.5, pp. 647-655, May, 2000.

[8] J. Tang, "The Shannon Channel Capacity of Dispersion-Free Nonlinear Optical Fiber Transmission", J. Lightwave Technol., vol. 19, no. 8, pp. 1104-1115, Aug. 2001.

[9] S.Wen, Y.Wang, W.Su, Y.Xiang, X.Fu and D.Fan, "Modulation instability in nonlinear negative-index material", Phys. Rev. E vol.73 no. 3. pp. 036617-, 2006.

[10] G. Agrawal, "Non linear Fiber Optics”, Ed. New York: Academic press, pp. 47, 62, 2013. 